\title{
Mechanisms in Adaptive Feedback Control: Photoisomerization in a Liquid
}

\author{
Kunihito Hoki and Paul Brumer \\ Chemical Physics Theory Group, Department of Chemistry, and Center for Quantum Information and Quantum Control, \\ University of Toronto, Toronto, Canada M5S $3 \mathrm{H6}$
}

(Received 13 December 2004; published 12 October 2005)

\begin{abstract}
The underlying mechanism for Adaptive Feedback Control in the experimental photoisomerization of 3,3'-diethyl-2,2'-thiacyanine iodide (NK88) in methanol is exposed theoretically. With given laboratory limitations on laser output, the complicated electric fields are shown to achieve their targets in qualitatively simple ways. Further, control over the cis population without laser limitations reveals an incoherent pump-dump scenario as the optimal isomerization strategy. In neither case are there substantial contributions from quantum multiple-path interference or from nuclear wave packet coherence. Environmentally induced decoherence is shown to justify the use of a simplified theoretical model.
\end{abstract}

DOI: 10.1103/PhysRevLett.95.168305

Experimental results on the quantum control of molecular processes [1,2] fall into two categories: those designed to explore the utility of a particular coherent control scenario, and those that use adaptive feedback [3-8] to attempt automated optimal control of a target process. The former have well understood mechanisms but are scenario specific, whereas the latter are generally applicable but have thus far provided very limited insight into the mechanisms by which control is achieved. Indeed, it is widely recognized that extracting the mechanism of control from an optimal control experiment is the central challenge in this research area [9]. In this Letter we (a) expose the simple mechanism underlying control in a recent [7] trans-cis isomerization control experiment, and (b) demonstrate that removing experimental restrictions on laser frequency and amplitude exposes modified versions of well known coherent control scenarios as the dominant control mechanism.

Gerber's seminal experiment [7] shows successful optimization of the trans to cis isomerization of 3,3'-diethyl2,2'-thiacyanine iodide (NK88) as well as suppression of the trans to cis transition, depending upon laser pulse shapes. As is typical of these experiments, the optimal pulse shapes in both cases are complex functions of phase, frequency, and time, and differ substantially for the two targets. Below we obtain quantitative agreement with these results. Further, we do so despite the complexity of the system, using a 1 degree of freedom model coupled to a bath. Indeed, it is precisely the presence of environmentally induced decoherence that simplifies the control problem.

Consider a model consisting of the system with Hamiltonian $H_{\mathrm{S}}$, the bath $H_{\mathrm{B}}$, system-bath coupling $H_{\mathrm{SB}}$, and system-electric field coupling described in the dipole approximation. The total Hamiltonian is given by

$$
H=H_{\mathrm{S}}+H_{\mathrm{B}}+H_{\mathrm{SB}}-\mu E(t),
$$

where $H_{\mathrm{S}}$ describes the isomerization process via a onedimensional reaction coordinate $\phi, H_{\mathrm{B}}$ represents all other degrees of freedom, $\mu$ is the transition dipole moment, and $E(t)$ is the incident electric field at time $t$.
PACS numbers: $82.50 . \mathrm{Nd}, 82.30 . \mathrm{Qt}, 82.53 . \mathrm{Uv}$

In terms of the two participating electronic states, the system Hamiltonian is given by

$$
H_{\mathrm{S}}=\left(\begin{array}{cc}
K+V_{\mathrm{g}}(\phi) & V_{\mathrm{ge}}(\phi) \\
V_{\mathrm{eg}}(\phi) & K+V_{\mathrm{e}}(\phi)
\end{array}\right),
$$

where $K=-\frac{\hbar^{2}}{2 m} \frac{\partial^{2}}{\partial \phi^{2}}$ is the kinetic energy, $V_{\mathrm{g}}(\phi)$ and $V_{\mathrm{e}}(\phi)$ are the ground and excited electronic state potential surfaces, and $V_{\mathrm{ge}}(\phi)=V_{\mathrm{eg}}(\phi)$ is the coupling potential between ground and excited states. In the adiabatic representation the ground state potential is a double well [7]. Populations reported below are of the eigenstates of the full molecular Hamiltonian $H_{\mathrm{S}}$. The simplest dynamics takes place by photoexcitation from the trans configuration to the excited electronic state followed by deexcitation to the cis and trans ground state via system-bath coupling.

In the case of 3,3'-diethyl-2,2'-thiacyanine iodide, the effective mass is $m=5 \mathrm{amu} \AA^{2}, V_{\mathrm{g}}(\phi)=A_{0}(1-\cos \phi)$, $V_{\mathrm{e}}(\phi)=A_{1}+A_{2} \cos \phi, V_{\mathrm{eg}}(\phi)=1000 \mathrm{~cm}^{-1}$, where $A_{0}$, $A_{1}$, and $A_{2}$ are $15900 \mathrm{~cm}^{-1}, \quad 17500 \mathrm{~cm}^{-1}$, and $7500 \mathrm{~cm}^{-1}$, respectively. These, and other parameters below were obtained by a fit to experimental results. The eigenvalues $\lambda_{i}$ and eigenvectors of the system are calculated by diagonalizing the molecular Hamiltonian matrix represented on a grid, where periodic boundary conditions at $2 \pi$ are imposed on the system. The transition dipole moment $\mu$ is expressed in terms of the two electronic states as a $2 \times 2$ dimensional matrix with zeroes on the diagonal, and off-diagonal elements $\mu_{\mathrm{ge}}(\phi)=10 \mathrm{D}$. This corresponds to the oscillator strength $f \simeq 1$ in the FranckCondon region of the trans configuration.

The bath is described as a set of harmonic oscillators of frequency $\omega_{\alpha}$ and the system-bath coupling is $H_{\mathrm{SB}}=$ $Q \sum_{\alpha} \hbar \kappa_{\alpha}\left(b_{\alpha}^{\dagger}+b_{\alpha}\right)$, where $b_{\alpha}^{\dagger}$ and $b_{\alpha}$ are the creation and annihilation operators pertaining to the $\alpha$ th harmonic oscillator. The operator $Q$ is a diagonal $2 \times 2$ matrix with $\cos \phi$ on the diagonal, and the coupling constant $\kappa_{\alpha}$ and spectrum of the bath are chosen in accord with an Ohmic spectral density $J(\omega)=2 \pi \sum_{\alpha} \kappa_{\alpha}^{2} \delta\left(\omega-\omega_{\alpha}\right)=\eta \omega e^{-\omega / \omega_{c}}$, where the strength of the system-bath coupling is deter- 
mined by the dimensionless parameter $\eta=5$, and $\omega_{c}=$ $450 \mathrm{~cm}^{-1}$. Given these parameters, the electronic dephasing time around the Franck-Condon region of the trans configuration is $\sim 10 \mathrm{fs}$, and virtually complete relaxation from excited trans to stable trans and cis occurs within $5 \mathrm{ps}$. The former is a typical characteristic dephasing time whereas the latter is chosen to agree with experiment.

The dissipative dynamics of the system was evaluated using the Redfield equation with secular approximation [10]. The evolution of diagonal elements $\rho_{i i}(t)$ of the system density matrix is given by

$$
\begin{aligned}
\frac{\partial}{\partial t} \rho_{i i}(t)= & -i \frac{E(t)}{\hbar} \sum_{m}\left[\rho_{i m}(t) \mu_{m i}-\mu_{i m} \rho_{m i}(t)\right] \\
& +\sum_{j \neq i} w_{i j} \rho_{j j}(t)-\rho_{i i}(t) \sum_{j \neq i} w_{j i},
\end{aligned}
$$

where the transition probability is $w_{j i}=\Gamma_{i j j i}^{+}+\Gamma_{i j j i}^{-}$and where each index denotes a state of the system, including the electronic and vibrational quantum numbers. Here, $\Gamma_{l j i k}^{-}=\left(\Gamma_{k i j l}^{+}\right)^{*}$ and

$$
\begin{aligned}
\Gamma_{k i j l}^{+}= & \frac{1}{2 \pi} Q_{l j} Q_{i k} \int_{0}^{\infty} d \tau \int_{0}^{\infty} d \omega J(\omega)\{[\bar{n}(\omega)+1] \\
& \left.\times e^{-i\left(\omega_{i k}+\omega\right) \tau}+\bar{n}(\omega) e^{-i\left(\omega_{i k}-\omega\right) \tau}\right\},
\end{aligned}
$$

where $\bar{n}(\omega)=\left\{\exp \left(\hbar \omega / k_{b} T\right)-1\right\}^{-1}$ is the Bose distribution, $T$ is a temperature, and $\omega_{j i}=\left(\lambda_{j}-\lambda_{i}\right) / \hbar$. After some algebra, we obtain

$$
w_{j i}=\left\{\begin{array}{ll}
\left|Q_{j i}\right|^{2} J\left(-\omega_{j i}\right)\left[\bar{n}\left(-\omega_{j i}\right)+1\right] & \text { for } \omega_{j i}<0 \\
\left|Q_{j i}\right|^{2} J\left(\omega_{j i}\right) \bar{n}\left(\omega_{j i}\right) & \text { for } \omega_{j i}>0
\end{array} .\right.
$$

The evolution of the off-diagonal elements is described as

$$
\begin{aligned}
\frac{\partial}{\partial t} \rho_{i j}(t)= & -i \omega_{i j} \rho_{i j}(t)-\gamma_{i j} \rho_{i j}(t) \\
& -i \frac{E(t)}{\hbar} \sum_{m}\left[\rho_{i m}(t) \mu_{m j}-\mu_{i m} \rho_{m j}(t)\right],
\end{aligned}
$$

with dephasing rate $\gamma_{i j}$ :

$$
\gamma_{i j}=\sum_{k}\left(\Gamma_{i k k i}^{+}+\Gamma_{j k k j}^{-}\right)-\Gamma_{j j i i}^{+}-\Gamma_{j j i i}^{-} .
$$

The resultant vibrational dephasing time within the excited electronic state is $\approx 15 \mathrm{fs}$.

To model the adaptive feedback experiment, the electric field comprises 128 frequency values, where the phases of each frequency component are the optimization parameters; the frequency width is $200 \mathrm{~cm}^{-1}$, and the time width is 2 ps. Specifically, the electric field function is therefore taken to be

$E(t)=\sum_{i=0}^{127} A \exp \left[-\left(\frac{t-t_{0}}{2 \Delta t}\right)^{2}-\left(\frac{\Omega_{i}-\Omega_{0}}{2 \Delta \Omega}\right)^{2}\right] \cos \left(\Omega_{i} t+\Theta_{i}\right)$,

where $A=5 \mathrm{MV} / \mathrm{m}, t_{0}=\Delta t=2 \mathrm{ps}, \Omega_{0}=25000 \mathrm{~cm}^{-1}$, $\Delta \Omega=200 \mathrm{~cm}^{-1}, \Omega_{i}=24800+3.125 i$ in $\mathrm{cm}^{-1}$, and $\Theta_{i}$ are the optimization parameters. Note that in accord with experiment [7], the field is optimized by varying the phases $\Theta_{i}$ using an evolutionary algorithm, and the field amplitude, as well as the overall frequency width of the pulse, are constrained. Further, the algorithm is designed to simulate experimental conditions [11], where the population size is $60 ; 10$ survivors are selected from the generation, each of which has 4 children by mutation and 1 child by crossover, and yielding again 60 individuals for the next generation.

In our second study, the experimental frequency and amplitude constraints were lifted and the optimal field was evaluated by using a monotonically convergent algorithm [12]. In both cases the optimization was carried out with a standard penalty on the laser power, and the initial condition $\rho_{i j}(0)$ was set to thermal equilibrium at temperature $T=300 \mathrm{~K}$. At this temperature the initial cis population is negligible.

Below, the time-dependent population of the stable trans, is defined as the projection of $\rho(t)$ onto the lowest 49 states localized around $\phi=0$ and the cis population as the lowest 23 states localized around $\phi=\pi$. The excited state population is the remainder.

Figure 1 shows the isomerization dynamics under the optimized electric field restricted in frequency and amplitude. In the experiment [7], the target was chosen as the ratio of the transmission change $\Delta T$ at $400 \mathrm{~nm}$ to that at $460 \mathrm{~nm}$, measured at $20 \mathrm{ps}$, assumed to be proportional to number of cis molecules created and number of trans molecules excited, respectively [13]. The target is modeled computationally as the ratio of population of stable cis to the laser pulse area, $\mu_{\mathrm{ge}} \int|E(t)| d t / \hbar$, at a target time $20 \mathrm{ps}$. Here the pulse area would be approximately proportional to the number of trans molecules excited, if the nuclei were fixed and two electronic states participated in the process. Its use corrects somewhat for the presence of decoherence in this one dimensional model, relative to the multidimensional decoherence-free excitation, experimentally. The electric field is optimized by 64 iterations from randomly initialized phases $\Theta_{i}$. The resultant electric field (Fig. 1), in accord with experiment, is seen to have a peak at $\sim 1.9 \mathrm{ps,}$ and is considerably sharper than the laser 2 ps envelope for each frequency component, implying that the optimization has yielded a nearly equal set of phases $\Theta_{i}$. Figure 1 also shows small peaks around the main peak, giving an overall structure that is almost quantitatively the same as the experimental result [7]. The bottom panel of Fig. 1 shows the isomerization dynamics; the system is seen to be excited by the electric field at $t \sim 2 \mathrm{ps}$, with maximum $P_{\mathrm{e}}(t) \simeq 0.4$. After photoexcitation, the system relaxes into stable trans and cis due to the system-bath coupling, giving a final cis probability of 0.17 . The optimized electric field gives a target value of 0.53 , while an unoptimized electric field gives a 0.7 times smaller value of 0.36 , in agreement with experiment.

Further studies shows that the small peaks in $E(t)$ are of little relevance. That is, after 200 iterations these small 


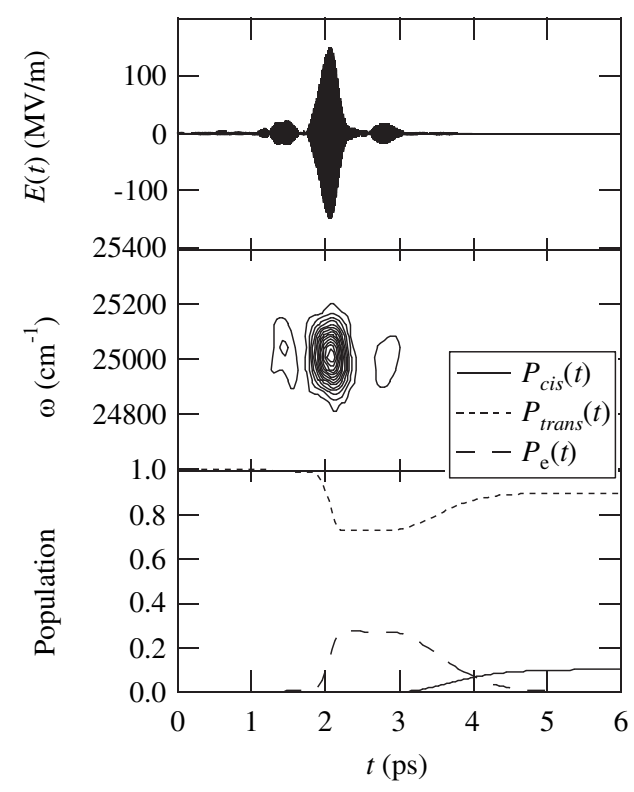

FIG. 1. Time evolution under the optimized electric field with restriction on frequency and amplitude. Top panel: an electric field obtained by 64 iterations from random-initial phases $\Theta_{i}$. Middle panel: time-frequency resolved spectrum. Bottom panel: time evolution of populations.

peaks almost disappear. Thus, the mechanism underlying the experimentally observed control is efficient photoexcitation under dissipative conditions, balancing the time scale for excitation and wave packet motion with the ongoing decoherence.

Experimental results were also presented [7] for the case of "no cis population," again using fields constrained in amplitude and frequency and using the transmission ratio as the target. The analogous computational result is shown in Fig. 2, in excellent agreement with experiment. Specifically, the pulse is now delocalized in time, rather complicated in form, and of duration longer than the dephasing time between the two electronic states. The maximum amplitude is considerably lower than that shown in Fig. 1. Examination of the populations (bottom panel, Fig. 2), shows that $P_{\mathrm{e}}(t)$ reaches only 0.006 , far lower than that in Fig. 1. Thus, despite the complexity of the pulse, its entire purpose is to ensure that there is no excitation of the initial trans species. However, the optimized electric field in our case gives a 0.05 target value, $\approx 4$ times smaller than the experiment, resulting from the fact that our model does not take into account competing processes such as excitations to other electronic states and the large number of nuclear vibrational modes.

The robustness of our results was checked by performing various alternate computations. For example, using the target "ratio of created cis to depleted trans" was not useful since this quantity is always unity at $20 \mathrm{ps}$. This is because in this model the molecule is, due to relaxation by $20 \mathrm{ps}$, either stable trans or stable cis. Choosing a slower relaxation rate, so that excited population remains at $20 \mathrm{ps,}$

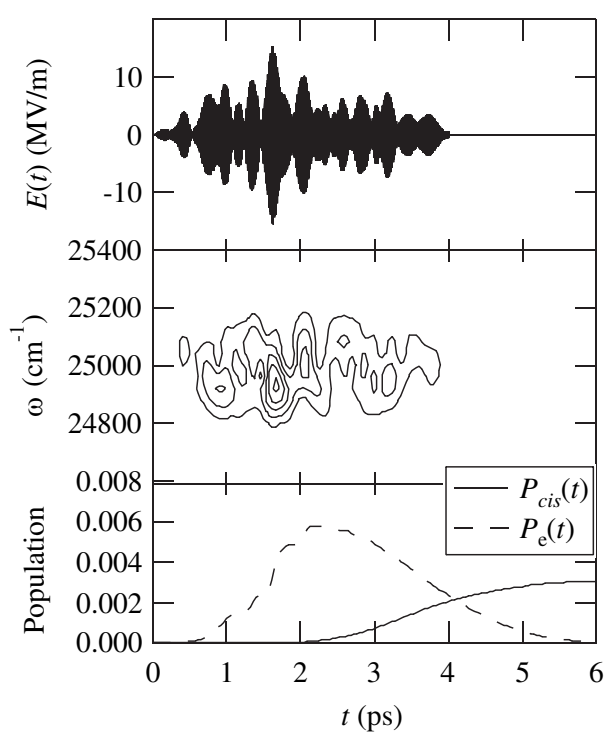

FIG. 2. Time evolution of the system under the optimized electric field that is restricted in frequency and amplitude. In this case, the target of the control is minimization of stable cis. Top panel: optimized electric field. Middle panel: time-frequency resolved spectrum. Bottom panel: time evolution of populations.

also did not improve the results. Here the target maximization showed an electric field moved to as early a time as possible to wait for the slow relaxation, or as late as possible in the case of minimization to avoid relaxation to the trans and cis molecule. Neither agree with experiment. However, using a simpler target, that is a population of stable cis, gives qualitatively the same fields as shown in Figs. 1 and 2, but dissimilar ratios of target improvement relative to the unmodulated pulse. Hence we are confident that the essential physics is contained in the simulation presented above.

The experimental results optimize the target within the restricted frequency range and intensity described above. Ideally, however, adaptive feedback control desires the $o p$ timal result, which would require unrestricted laser equipment. To examine one such optimal solution we repeated the adaptive feedback studies with penalties on the power, but with no frequency restriction on the laser and with the population of stable cis used directly as the target.

In the second of these target cases, the suppression of trans to cis isomerization, the optimal solution obtained was $E(t)=0$, i.e., no excitation. By contrast, the fully optimal pulse for producing cis from trans is shown in Fig. 3 where, for computational convenience, the target time is $5 \mathrm{ps}$. The optimal electric field seen to consist of a pump pulse (from 0 to $0.01 \mathrm{ps}$ ), and dump pulses (from 0.05 to $0.13 \mathrm{ps}$ ). The dump pulse has several frequency components, and is resonant with the deexcitation between electronic states around $\phi=2.1 \mathrm{rad}$. After the pump pulse, $P_{\mathrm{e}}(t)$ reaches almost 1 , and after the dump pulse, $\sim 0.1$ of population is transferred from $P_{\mathrm{e}}(t)$ to $P_{\text {cis }}(t)$. Subsequently (not shown), the remaining excited component relaxes due to the system-bath coupling giving a final 


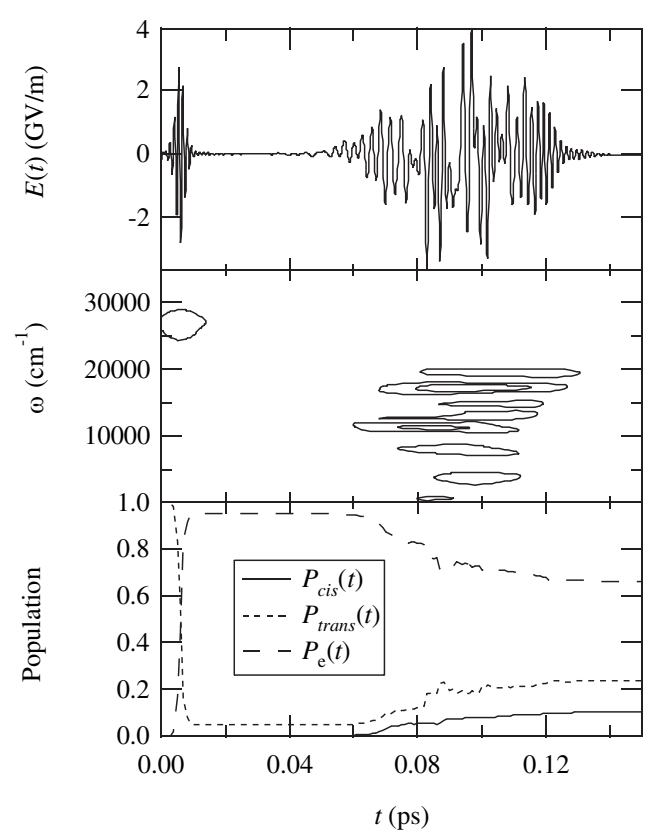

FIG. 3. Short-time evolution of the system under the fully optimized pulse. After $0.15 \mathrm{ps}$, the electric field is essentially zero. Middle panel: time-frequency resolved spectrum of the pulse. Bottom panel: time evolution of populations.

cis probability of 0.36 , far higher than the cis probability obtained when this computation was repeated with the laser frequency restrictions above.

Note that unlike the paradigmatic pump-dump coherent control scenario $[1,14]$, or the previously proposed eigenstate based cis-trans isomerization mechanism [15], the pump and dump steps are not coherently related since the system is decohered between pulses. Hence the optimal mechanism in this case is desirable excitation of an excited state population, delocalizing and decohering of the excited state wave packet, and desirable deexcitation of the decohered excited state population. The delay between the pump and dump steps results from the spreading of density into the cis region.

It should be noted that the optimal pulse has almost zero amplitude after $0.15 \mathrm{ps}$, since it is very difficult to try to make more cis after the dump process. That is, a second photoexcitation step from the stable trans to the excited state is not useful, since the excited component cannot relax to stable cis within the few allotted ps. Also, due to the dephasing of the system, the excited component spreads rapidly into whole $2 \pi$ range of $\phi$. Any attempt at a second photo-deexcitation from these excited components to stable cis would also be ineffective since it would be accompanied by compensatory excitation from the populated $c i s$ to the excited state.

In summary, this work has (a) successfully exposed the simple underlying mechanisms associated with the complex experimental results of an adaptive, condensed phase, feedback experiment; (b) demonstrated the role of fre- quency limitations in the experimental laser wavelengths and the concomitant emergence of an incoherent pumpdump scenario when these restrictions are lifted, with cis population as the target; and (c) demonstrated the utility of the simplest of models, one-dimensional motion plus decoherence. The utility of such models derives from the fact that the decoherence is fast and that the measurement of isomer identity implicitly ignores all degrees of freedom but one (the angle $\phi$ ). Hence, one-dimensional motion plus decoherence is formally the proper description. Note, however, that an appropriate representation of the decoherence is necessary to achieve the quality of results shown here. Since most liquid phase control experiments will be of a similar nature, simple control models of this type may be justified generally by the role and presence of decoherence. However, if decoherence is slower than the characteristic molecular dynamics then the problem is more complex and dynamics in many degrees of freedom must be explicitly controlled.

We thank Professor Gustav Gerber, Dr. Gerhard Vogt, and Dr. Gerhard Krampert for discussions on their experiment. This work has been supported by Photonics Research Ontario and the Natural Sciences and Engineering Research Council of Canada.

[1] M. Shapiro and P. Brumer, Principles of the Quantum Control of Molecular Processes (Wiley, New York, 2003).

[2] M. Dantus and V. V. Lozovoy, Chem. Rev. 104, 1813 (2004).

[3] R.S. Judson and H. Rabitz, Phys. Rev. Lett. 68, 1500 (1992).

[4] C. J. Bardeen, V. V. Yakovlev, K. R. Wilson, S.D. Carpenter, P. M. Weber, and W. S. Warren, Chem. Phys. Lett. 280, 151 (1997).

[5] R. J. Levis, G. M. Menkir, and H. Rabitz, Science 292, 709 (2001).

[6] T. Brixner and G. Gerber, Chem. Phys. Chem. 4, 418 (2003).

[7] G. Vogt, G. Krampert, P. Niklaus, P. Nuernberger, and G. Gerber, Phys. Rev. Lett. 94, 068305 (2005).

[8] B. J. Pearson and P. H. Bucksbaum, Phys. Rev. Lett. 92, 243003 (2004); 94, 209901 (2005).

[9] C. Daniel, J. Full, L. González, C. Lupulescu, J. Manz, A. Merli, S. Vajda, and L. Wöste, Science 299, 536 (2003).

[10] K. Blum, Density Matrix Theory and Applications (Plenum, New York, 1981).

[11] T. Baumert, T. Brixner, V. Seyfried, M. Strehle, and G. Gerber, Appl. Phys. B 65, 779 (1997).

[12] Y. Ohtsuki, W. S. Zhu, and H. Rabitz, J. Chem. Phys. 110, 9825 (1999).

[13] G. Vogt and G. Krampert (private communication).

[14] D. J. Tannor and S. A. Rice, J. Chem. Phys. 83, 5013 (1985).

[15] D. Gruner, P. Brumer, and M. Shapiro, J. Phys. Chem. 96, 281 (1992). 